\title{
A Model for Play-based Intervention for Children with ADHD
}

\author{
Reinie Cordier \\ University of Sydney, Faculty of Health Sciences, Australia
}

Introduction: The importance of play has been described by many play authors (Parham, 2008). Even though children with ADHD experience serious social problems, there is limited research on their play. By integrating literature on ADHD with literature on play, we can postulate how play is influenced by the characteristics of ADHD. These postulations enabled us to propose a theoretical model (proposed model) to depict the interactive process between the characteristics of ADHD and factors that promote play.

Objectives: The purpose of this paper is to present the revised model as it was adjusted based on our findings and to present preliminary principles for play-based interventions for children with ADHD derived from our findings.

Methods: We tested the proposed model in a study comparing two groups of children $(n=350)$ between the ages of 5 and 11 years. One group consisted of children diagnosed with ADHD $(n=112)$ paired with playmates $(n=112)$ who were typically developing; the control group consisted of typicallydeveloping children paired with typically-developing playmates $(n=126)$. The Test of Playfulness (ToP) was administered and the model was revised in line with the findings.

Results: None of the ToP items that related directly to the primary symptoms of ADHD differed significantly between children with ADHD and typically-developing children, suggesting that the primary symptoms of ADHD (inattention, hyperactivity and impulsivity) did not account for the difficulties experienced by the children with ADHD with regard to play. The findings suggest difficulties in the social play and lack of interpersonal empathy in the play of children with ADHD.

Conclusions: We draw on the revised model to propose preliminary principles for play-based interventions for children with ADHD.

Contribution to the practice/evidence base of occupational therapy: The principles emphasise the importance of capturing the motivation of children with ADHD, counteracting the effects of lack of interpersonal empathy, and considerations for including playmates in the intervention process.

References:

Parham, L. D. (2008). Play and occupational therapy. In L. D. Parham \& L. S. Fazio (Eds.), Play in occupational therapy for children (2nd ed., pp. 3-42). St. Louis: Mosby. 